\title{
Fractal analysis of damage detected by acoustic emissions in prestressed reinforced concrete beam under loading
}

\author{
Wang You-Shun ${ }^{1}$, Liu Jian ${ }^{1}$ \\ (1 Zhumadian Highway Administration Bureau , Zhumadian 463000 ,Henan, China)
}

Keywords: acoustic emission: prestressed reinforced concrete: fractal: relevant dimension Abstract. For researching the relationship between prestressed reinforced concrete acoustic emissions (AE) signal and its damage evolution information, the paper studies AE signal parameter sequence fractal characteristics for destruction of whole process of prestressed reinforced concrete under different stress level. The research results show that in the process of destruction, AE signal energy parameter sequence has obvious fractal characters at a scale. AE process has different degree of self-similarity under different loading level, so the mechanical state beam structure can be described by the correlation dimension. It indicated that beam structure has damage when correlation dimension cuts down to a minimum and then reaches to the maximum. This model of the range of the changes reflects the damage degree. It will appear more serious damage when the correlation dimension is in stable state after damage. It gives the theoretical basis and methods for prestressed concrete structure health monitoring and nondestructive testing.

\section{Introduction}

Acoustic emission(AE) technique is a new kind of health monitoring and nondestructive testing technology. When the concrete structure under the effect of local stress or internal force, the transient energy of deformation or crack propagation was released in the form of elastic wave, this phenomenon was called acoustic emission phenomenon ${ }^{[1]}$. According to the analysis of the collected information, we can deduce the process of structural damage. In practical application due to the concrete itself is an open 、 energy consumption and the nonlinear system with chaos characteristic, coupled with the complexity of its working environment, all that often cause more actual monitoring signal interference, covers some key information. Fractal theory is developing rapidly in recent years, and become outshine others in the nonlinear science, fractal theory has been widely used in social science and natural science ${ }^{[2]}$. In the field of civil engineering, fractal theory has been applied to the study of concrete materials and components by some scholars ${ }^{[2-5]}$.

This paper starting from the structural health monitoring and nondestructive testing purpose, which apply fractal theory to analysis of acoustic emission phenomenon of concrete structure, and make the acoustic emission characteristic parameters of correlation dimension model. The experiment can prove that the existence of prestressed reinforced concrete AE phenomenon of fractal characteristics, and obtain he law of fractal characteristics correlation dimension change in the process of prestressed reinforced concrete damage. We can link the acoustic emission signal with damage stage of prestressed concrete in order to obtain more nature information of reinforced concrete damage. All that can provide the theory basis of accurately for concrete structure health monitoring and nondestructive testing.

\section{Acoustic emission experiment}

\section{Experiment introduction}

In the experiment we make four scale model of prestressed reinforced concrete beams, cross section size: $200 \mathrm{~mm} \times 250 \mathrm{~mm}$,span: $1200 \mathrm{~mm}$, steel bar arrangement, strength grade of concreteC 40 .

we adopts the industrial science and technology Beijing sound SWAEU2S acoustic emission instrument for acoustic emission device, the instrument adopts the four $150 \mathrm{KHZ}$ piezoelectric sensor. Sensors in the same span $200 \mathrm{~mm}$ linear beam side. 


\section{loading method}

The experiment adopts hierarchical load method which is divided into 11 levels and every level includes some stages. At various levels in different stages of the naming of the $\mathrm{ij}$, the $\mathrm{i}$ is mainly level such as $1 \mathrm{j}, 2 \mathrm{j}, 3 \mathrm{j}$, respectively loading force $1,2,3$ levels of load, $\mathrm{j}$ is the $\mathrm{j}$ stage in the $\mathrm{i}$ level. i.e. the meaning of 54 is the 4 stage in the 5 level, the maximum load power to achieve 5 level, the loading force is equal to 4 small stage one of the most strongly (i.e., $21.49 \mathrm{kN}$ ); If $\mathrm{j}=\mathrm{I}+1$, said at the corresponding level load unload stages such as 45, 56, 67. All levels of the residual are shown in table 1 below.

\begin{tabular}{cc}
\hline Load levels & Load/kN \\
\hline 1 & 3.364 \\
2 & 9.40 \\
3 & 15.44 \\
4 & 21.49 \\
5 & 27.53 \\
6 (yield load) & 33.57 \\
7 & 39.61 \\
8 & 45.65 \\
9 & 54.34 \\
10 & 63.04 \\
11 (ultimate load) & 71.73 \\
\hline
\end{tabular}

Table2 The max loads of every stage

\section{The AE signal parameter fractal dimension model}

Fractal dimension is the basic described fractal theory, the correlation dimension is one of relatively commonly fractal dimension. In this paper, based on $\mathrm{G}-\mathrm{P}$ algorithm to establish correlation dimension model, take the acoustic emission basic parameters sequence as the research object, basic parameters of each acoustic emission sequence corresponding to a set of capacity of the sequence of $n$ [4]:

$$
X=\left\{x_{1}, x_{2}, \ldots ., x_{n}\right\}
$$

$m$ dimensional phase space consists of $\operatorname{eq}(1)(m<n)$. First, take the former number $m$ as a vector of $m$ dimensional space

$$
X_{1}=\left\{x_{1}, x_{2}, \ldots ., x_{m}\right\}
$$

Then back again to take a number $\mathrm{m}$ number, constitute the second vector

$$
X_{2}=\left\{x_{2}, x_{3}, \ldots ., x_{m+1}\right\}
$$

By analogy, can form $\mathrm{N}=\mathrm{N}-\mathrm{m}+1$ vector.

Associated function is defined as:

$$
W[r(k)]=\frac{1}{N^{2}} \sum_{\substack{i=1, j=1 \\ i \neq j}}^{N} \theta\left(r-\left|X_{i}-Y_{j}\right|\right)
$$

eq (4) $\theta$ as the Heaviside function, the $r$ for a given scale, meaning the distance between two points of fractal, in order to avoid dispersion take $r$ as:

$$
r(k)=k \frac{1}{N^{2}} \sum_{\substack{i=1, j=1 \\ i \neq j}}^{N} \theta\left(r-\left|X_{i}-Y_{j}\right|\right)
$$


If there is a constant $\mathrm{D}$ in the associated function $\mathrm{W}(\mathrm{r}(\mathrm{k}))$ to obey the following relations, essentially it portrays the phase space of the self-similarity.

$$
\lim _{r \rightarrow 0} W(r(k)) \infty r^{D}
$$

$\mathrm{D}$ is called the correlation dimension, the fractal characteristics of phase space point at this time. Correlation dimension $\mathrm{D}$ as follows:

$$
D=\lim _{r \rightarrow 0} \frac{\ln W(r(k))}{\ln r}
$$

So drawing in logarithmic coordinates points $(\ln \mathrm{W}(\mathrm{r}(\mathrm{k}))$, $\mathrm{LNR}(\mathrm{k}))$ under a given scale $\mathrm{r}(\mathrm{k}), \mathrm{k}=1,2$, $3 \ldots \mathrm{g}$. Then fitting the number of point data, if the fitting result is linear, which suggests that under the given scale of the acoustic emission signal parameters have fractal characteristics. These points to synthesis of linear slope are correlation dimension D.

\section{Prestressed reinforced concrete of different loading stages AE energy characteristic parameter of fractal analysis}

According to the test process of beam crack and fracture development situation ,the failure process of prestressed reinforced concrete damage process can be divided into four stages under 3 11load level condition ( $1 \sim 2$ load level beams in the elastic stage and few AE signals) : I stage, tiny crack are forming and expanding Inside the concrete ( $3 \sim 4$ load level); II stage, Macro tiny crack are forming on surface (5 7load level); III stage , crack are forming and expanding through surface (8 10load level); IV stage, Macroscopic Crack fracture are forming and expanding (11). Calculation the four stages of $\mathrm{AE}$ energy parameters of loading sequence correlation dimension at all levels by computing.

Found by calculation, the I stage due to the constraint of reinforcing steel bar and steel strand for concrete, which makes the correlation dimension is larger in the early. As the load from 3 level to 4 level correlation dimension declined, which means that the internal micro cracks of concrete gradually transition from disorder to the order and small crack to the macro crack development. In II stage the51、52 two small stage because of the influence of the Kaiser [8], no obvious AE signal and loading to 54, 55 stages appeared obvious AE signal, the 54, 55 two stage correlation dimension only small changes and smaller compared with I stage. Under6 $\sim 7$ load level correlation dimension presents a trend of local changed repeatedly, but the overall downward trend, the internal micro cracks of concrete appear constantly and collect, then form macroscopically surface tiny cracks, the damage process are interlaced together. III stage correlation dimension of each stage repeated change bigger than in II stage, this time the damage of concrete are more order and mostly is surface crack, which from small scale to large scale events. IV stage under 11load level 119, 1110, 1111 small stage, correlation dimension approximately trends to be stable and shows that the beam concrete surface damage has been basically stable, cracks on the surface development to deeper. when load reach to the 1111 stage beam cross appear crack fracture clearly. Under various load correlation dimension calculation II, III, IV stage correlation dimension with load (F/Fultimate) change trend diagram as shown in figure 5. 

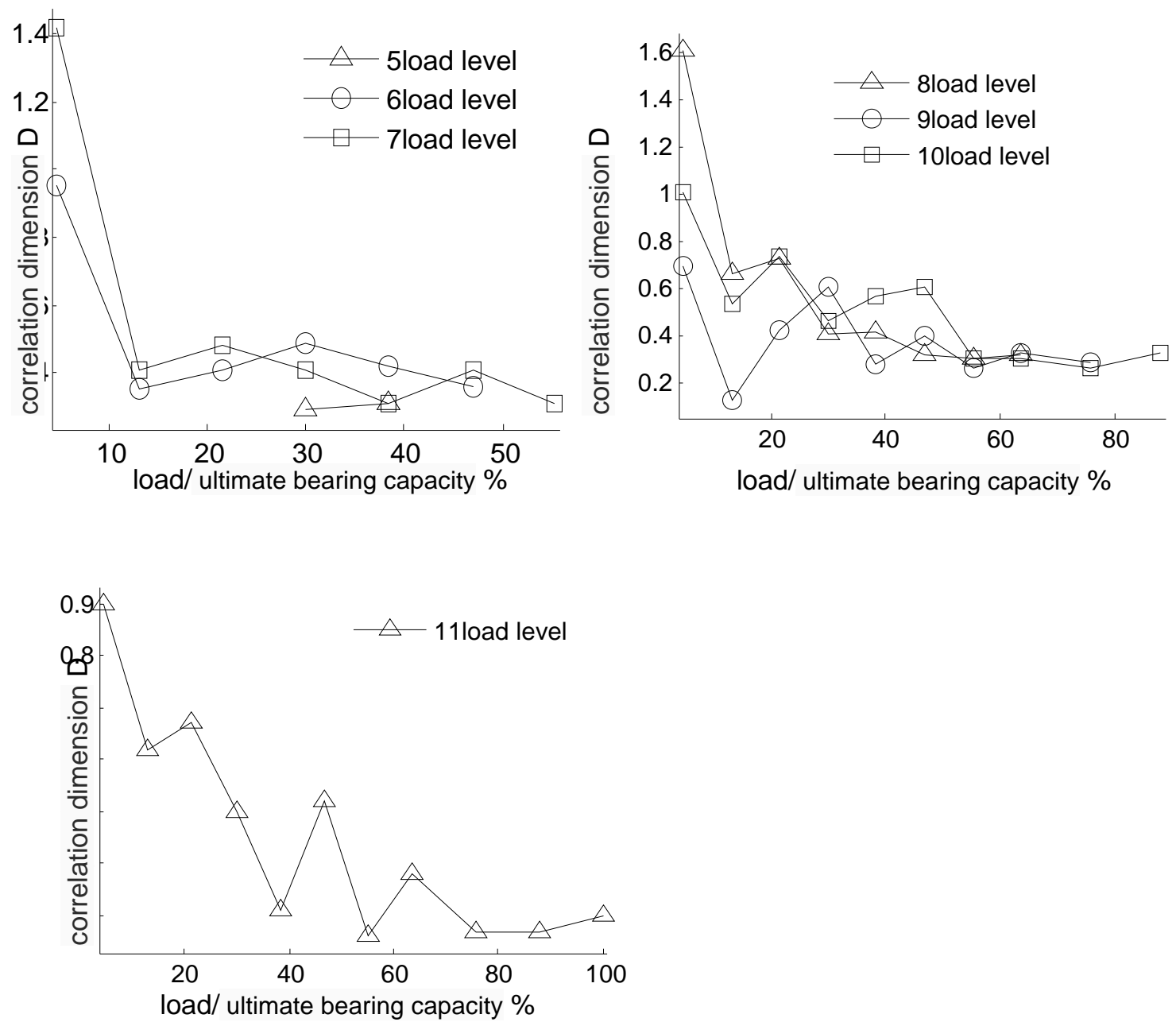

Fig.1 Correlation dimension curve distribution under II、III、IVstage

From the three figure can be seen in the picture with the load increasing the magnitude of the correlation dimension change is more and more bigger, damage from micro cracks to cracks. Correlation dimension appear this pattern down $\rightarrow$ minimum $\rightarrow$ up, Which means that the concrete damage will occur, the magnitude of the correlation dimension change reflect the degree of damage. By the above discussed, the correlation dimension change pattern can provide scientific theory basis for the bridge structural health monitoring and nondestructive testing.

\section{Conclusion}

(1) The energy correlation dimension appear the pattern down $\rightarrow$ minimum $\rightarrow$ up that can be regard as a sign of prestressed concrete beam structure damage, at the same time, this model reflect the degree of damage.

(2)In the wake of certain damage subsequent level of correlation dimension is in stable state, meaning the prestressed reinforced concrete beam will appear larger damage, some measures should focus on monitoring or repairing

\section{References}

[1] Ge R D,Liu M J,Lv H B, Experimental research on acoustic characteristics of reinforced concrete beams during failure process [J].Journal of Guangxi University(Natural Science Edition) 201136 (1) : 160-165

[2] Alireza A, Seyed S, Farshad R. Quantifying the effects of crack width, tortuosity, and roughness on water permeability of cracked mortars [J]. Cement and Concrete Research, 2012, 42(2): 313-320. 
[3] Cao M S, Ren QW, Zhuo A L Experimental study on fractal characterization in damages of concrete structures [J]. Rock mechanics, 200526 (10) 1570-1574

[4] Ji H G, Wang J C, Shan XY,etal Fractal charcteristics of AE process of concrete material and application to the fracture analyses[J].Chinese Journal of Rock mechanics and Engineering, 2001 20(6):; 801-804

[5] Savas E, Marva A B. Fractal-fracture analysis and characterization of impact-fractured surfaces in different types of concrete using digital image analysis and 3D nanomap laser profilometery [J]. Construction and Building Materials, 2013, 40: 70-76.

[6] Fu Q,Li C X,Zhang C X ,G-P algorithm for evaluating the correlation dimension in chaos [J].Journal of PLA University of Science and Technology(Natural Science Edition)，2014 15（3） 275-282

[7] Wang X Q,Ge H K,etal,Experimental studyof two types of sample acoustic emission events and Kaiser effect point recognition approach[J]. Chinese Journal of Rock mechanics and Engineering, 201130 (3) 580-587

[8] Wu S X, Zhang S X, Shen D J, An experimental study on Kaiser effect of acousticemission in concrete under uniaxial tensionloading[J].Chinses Civil Engineering Journal. 2008 41 (4) 32-38 